\title{
Network Structure Shapes Spontaneous Functional Connectivity Dynamics
}

\author{
Kelly Shen, ${ }^{1}$ R. Matthew Hutchison, ${ }^{2,4}$ Gleb Bezgin, ${ }^{1}$ Stefan Everling, ${ }^{2,3}$ and Anthony R. McIntosh ${ }^{1,5}$ \\ ${ }^{1}$ Rotman Research Institute, Baycrest, Toronto, Ontario M6A 2E1, Canada, ${ }^{2}$ Robarts Research Institute and ${ }^{3}$ Department of Physiology and Pharmacology, \\ University of Western Ontario, London, Ontario N6A 5K8, Canada, ${ }^{4}$ Harvard University, Department of Psychology, Center for Brain Science, Cambridge, \\ Massachusetts 02138 , and ${ }^{5}$ Department of Psychology, University of Toronto, Toronto, Ontario M5S 3G3, Canada
}

\begin{abstract}
The structural organization of the brain constrains the range of interactions between different regions and shapes ongoing information processing. Therefore, it is expected that large-scale dynamic functional connectivity (FC) patterns, a surrogate measure of coordination between brain regions, will be closely tied to the fiber pathways that form the underlying structural network. Here, we empirically examined the influence of network structure on FC dynamics by comparing resting-state FC (rsFC) obtained using BOLD-fMRI in macaques (Macaca fascicularis) to structural connectivity derived from macaque axonal tract tracing studies. Consistent with predictions from simulation studies, the correspondence between rsFC and structural connectivity increased as the sample duration increased. Regions with reciprocal structural connections showed the most stable rsFC across time. The data suggest that the transient nature of FC is in part dependent on direct underlying structural connections, but also that dynamic coordination can occur via polysynaptic pathways. Temporal stability was found to be dependent on structural topology, with functional connections within the rich-club core exhibiting the greatest stability over time. We discuss these findings in light of highly variable functional hubs. The results further elucidate how large-scale dynamic functional coordination exists within a fixed structural architecture.
\end{abstract}

Key words: functional connectivity; functional MRI; large-scale dynamics; rich club organization; structural connectivity

\section{Introduction}

The importance of ongoing fluctuations in neuronal systems to support behavior has long been recognized (Pinneo, 1966; Rabinovich et al., 2012) and a growing number of studies are using BOLD-fMRI as a method to explore these intrinsic dynamics. Functional connectivity (FC) varies significantly across timescales, even in the absence of an explicit task (Hutchison et al., 2013a). Resting-state FC (rsFC) dynamics are thought to reflect the brain's exploration of different network configurations. These configurations may be attributed to the diverse set of potential cognitive processes that unfold in an unconstrained manner over the course of a resting-state scan (Bressler and Kelso, 2001; Deco et al., 2013). Regional differences in rsFC dynamics exist, in which interactions involving certain regions can be more variable over time (Honey et al., 2007; Gonzalez-Castillo et al., 2014). Interestingly, those same regions are often described as "hubs," densely connected regions that are topologically positioned to serve flexible and integrative roles across different functional subnetworks (van den Heuvel and Sporns, 2013a).

\footnotetext{
Received Dec. 2, 2014; revised Feb. 17, 2015; accepted Feb. $26,2015$.

Author contributions: K.S., R.M.H., S.E., and A.R.M. designed research; R.M.H. and S.E. performed research; K.S. and G.B. analyzed data; K.S., R.M.H., and A.R.M. wrote the paper.

This work was supported by the J.S. McDonnell Foundation (A.R.M.) and the Canadian Institutes of Health Research (CIHR) (S.E.). K.S. and R.M.H. hold Fellowship Awards from the CIHR.

The authors declare no competing financial interests.

Correspondence should be addressed to K. Shen, Rotman Research Institute, Baycrest, 3560 Bathurst Street, Toronto, ON M6A 2E1, Canada. E-mail: kshen@research.baycrest.org.

DOI:10.1523/JNEUROSCI.4903-14.2015

Copyright $\odot 2015$ the authors $\quad 0270-6474 / 15 / 355579-10 \$ 15.00 / 0$
}

Anatomical connectivity has been shown to constrain functional networks constructed at coarse timescales with implications for both local and global brain communication. Locally, functionally connected regions tend to be structurally connected (Greicius et al., 2009; van den Heuvel et al., 2009) and the strength of a functional connection depends in part on the density of its underlying white matter tracts (Hagmann et al., 2008; Hermundstad et al., 2013). FC is also mediated by polysynaptic connections, as rsFC exists between regions lacking direct structural connections (Adachi et al., 2012). More globally, structural constraints dictate the modular organization of functional networks. Dense bidirectional anatomical connectivity supports functional specialization within modules, whereas sparse unidirectional patterns are associated with network segregation into different functional modules (Shen et al., 2012). Intermodular communication is possibly mediated by a set of hubs that are densely structurally connected to one another, a "rich club" that forms a strong anatomical core (van den Heuvel and Sporns, 2013 b). Together, these findings suggest that the neuroanatomical architecture plays an important role in shaping the "static" organization of intrinsic functional networks.

Theoretical studies have suggested that rsFC dynamics emerge from a fixed anatomical architecture via differences in intrinsic dynamics of the individual node (Deco et al., 2011; Hansen et al., 2015). In these models, anatomical connectivity is best reflected by rsFC at coarse timescales and is only weakly correlated with rsFC at finer timescales (Honey et al., 2007). The observation that hubs are functionally variable (Honey et al., 2007; Mišić et al., 2011), especially 
with each other (Allen et al., 2014), seems contradictory to the evidence that hubs are densely structurally interconnected. Presumably, these connections should mostly limit the functional interactions of hubs to other hubs. In this study, we examined the extent to which network structure may constrain the moment-to-moment fluctuations in rsFC at both the local and global level. Specifically, we tested the hypotheses that local anatomical connectivity confers stability to functional connections and that the brain's structural topology constrains the variability of functional connections.

\section{Materials and Methods}

Animal preparation, data acquisition, image preprocessing, and the anatomical dataset have been described previously in detail (Hutchison et al., 2011; Bezgin et al., 2012; Shen et al., 2012) and are briefly outlined below.

Macaque fMRI. fMRI data were obtained from 6 (4 female) adult macaque monkeys (Macaca fascicularis, $3.6-5.3 \mathrm{~kg}$, age $5.10 \pm 0.48$ years). All surgical and experimental protocols were approved by the Animal Use Subcommittee of the University of Western Ontario Council on Animal Care and were in accordance with the Canadian Council on Animal Care guidelines.

Animals were anesthetized before their scanning session and anesthesia was maintained using $1 \%$ isoflurane ( 0.78 minimum alveolar concentration) (Tinker et al., 1977) in oxygen during image acquisition. Isoflurane is a commonly used anesthetic in resting-state investigations of nonhuman primates, revealing robust and homologous FC patterns (Vincent et al., 2007; Shmuel and Leopold, 2008; Teichert et al., 2010; Hutchison et al., 2011; Mars et al., 2011; for review, see Hutchison and Everling, 2012) while eliminating training requirements, physiological stress, and motion. At higher dosages, isoflurane has cooccurring effects on cerebral blood flow (CBF), blood volume, and metabolic rate that can result in neurovascular decoupling-confounding observations of neural changes (Masamoto and Kanno, 2012). However, the level used in the present study has been shown to preserve CBF autoregulation (Eger, 1984; Li et al., 2013) and result in slow continuous EEG activity and not burst suppression, a pattern characteristic of isoflurane at higher dosages (Vincent et al., 2007). Dose-dependent analysis has shown stable spatial and temporal FC patterns at doses $<1.50 \%$, suggesting a preservation of intrinsic functional organization (Hutchison et al., 2014).

Images were acquired using a $7 \mathrm{~T}$ Varian scanner and a Siemens AC84 gradient subsystem with an in-house designed and manufactured fivechannel primate head RF coil. For each monkey, two runs of 300 continuous echoplanar imaging (EPI) functional volumes were acquired (TR = $2000 \mathrm{~ms}, \mathrm{TE}=16 \mathrm{~ms}$, flip angle $=70^{\circ}$, slices $=30$, matrix $72 \times 72$, FOV $=96 \mathrm{~mm}$, voxel size $=1.3 \times 1.3 \times 1.5 \mathrm{~mm}$ ). The duration of each scan was $10 \mathrm{~min}$. High-resolution $\mathrm{T}_{2}$-weighted anatomical images were also acquired for each monkey $(\mathrm{TR}=5000 \mathrm{~ms}, \mathrm{TE}=38.6 \mathrm{~ms}$, echo train length $=5$, effective echo $=3$, slices $=30$, matrix $256 \times 250$, FOV $=96$ $\mathrm{mm}$, voxel size $=375 \mu \mathrm{m} \times 384 \mu \mathrm{m} \times 1.5 \mathrm{~mm}$ ).

Functional image preprocessing was performed using the FMRIB Software Library (FSL; http://www.fmrib.ox.ac.uk) and included motion correction, spatial smoothing (FWHM $=3 \mathrm{~mm}$ ), high-pass temporal filtering (Gaussian-weighted least-squares straight line fitting, $\sigma=$ $100 \mathrm{~s}$ ), low-pass temporal filtering (Gaussian filter, HWHM = 2.8 s), and brain extraction for normalization to the F99 atlas template (Van Essen, 2004; http://sumsdb.wustl.edu/sums/macaquemore.do). Nuisance variables were removed by regression using the Analysis of Functional NeuroImages (AFNI) software package (http://afni.nimh.nih.gov/afni). These included six motion parameters, as well as the global white matter and CSF signals. Regression of the global mean signal was not performed.

Anatomical dataset. The anatomical dataset was derived from the CoCoMac database (Stephan et al., 2001; Bakker et al., 2012; http://cocomac.g-node.org) and specified as connectivity between 82 cortical regions of interest (ROIs; 41 per hemisphere) based on the Regional Map parcellation of Kötter and Wanke (2005). The binary structural connectivity matrix therefore indicated the presence or absence of interareal axonal projections previously reported in tract tracing studies in macaques. We treated connections for which there was no information in CoCoMac the same as connections for which there was an explicit statement of absence of connectivity. For intrahemispheric connectivity, only a small proportion of connections were unknown (L: 192/1406, 13.7\%; R: 202/1406, 14.4\%). For interhemispheric connectivity, a greater proportion of connections were unknown $(882 / 1406,62.7 \%$ for each interhemispheric direction). Limiting data analyses to only explicitly present/absent connections produced qualitatively similar results.

The structural connectivity matrix has been described previously in detail (Bezgin et al., 2012). Briefly, the intrahemispheric connectivity matrix densities were 0.74 and 0.73 for right and left hemispheres, respectively. The high density of our structural matrix is likely due to the coarseness of the RM parcellation. Finer parcellations of anatomical connectivity based on CoCoMac data have been reported to have significantly lower density (Modha and Singh, 2010). The proportion of unidirectional intrahemispheric connections was 0.16 and 0.14 for the right and left hemispheres, respectively. These values match well with a recent rigorous histological description of macaque intrahemispheric connectivity (Markov et al., 2014). Our parcellation provides whole cortical coverage including interhemispheric connections.

The Regional Map parcellation was drawn on the F99 macaque standard cortical surface template (Van Essen et al., 2001) and transformed to voxel space with a $2 \mathrm{~mm}$ extrusion using the Caret software package (http://www.nitrc.org/projects/caret/; Bezgin et al., 2012).

Data analysis. ROIs for the fMRI data were defined using the Regional Map parcellation in F99 voxel space. tSNR maps were visualized to ensure BOLD signal quality was consistent across the cortex. Six ROIs in the ventral temporal region (three per hemisphere) were not included in the analyses for this study because EPI coverage did not extend to these regions in two animals. These were the bilateral amygdala, bilateral inferior temporal cortex, and bilateral temporal polar cortex. The structural connectivity matrix was adjusted accordingly and a total of 76 ROIs were included in the analyses (Fig. 1A, Table 1). For each fMRI scan, a weighted average time series was calculated for each ROI using a probabilistic weighting scheme.

"Static" rsFC for each scan was derived using regionwise Pearson correlations on whole time series (600 s or 300 volumes; Fig. 1B, top). "Dynamic" rsFC was measured using a sliding window approach: Regionwise Pearson correlations were performed on truncated time series of $30 \mathrm{~s}$ (15 volumes), $44 \mathrm{~s}$ ( 22 volumes), $60 \mathrm{~s}$ (30 volumes), $120 \mathrm{~s}$ (60 volumes), and $240 \mathrm{~s}$ (120 volumes; Fig. $1 \mathrm{~B}$, bottom). The window was advanced in increments of one time point along the entire time series and the correlation recalculated. Window sizes were selected based on previous studies of rsFC dynamics (Hutchison et al., 2013b; Allen et al., 2014). Each FC matrix was corrected for multiple comparisons using a false discovery rate (FDR) procedure (Benjamini et al., 2006) with the FDR set to $p<0.05$. If regionwise correlation $p$-values did not reach the FDR criterion, those elements of the FC matrix were set to zero.

To describe the correspondence between structural connectivity and the FC networks derived from both the static and dynamic conditions, we computed their cosine similarity by first reshaping each FC matrix and the structural connectivity matrix into vectors (Shen et al., 2012). Akin to a correlation, similarity can range from -1 to 1 . Values approaching 1 indicate that the vectors are highly alike, or correlated, and those approaching -1 indicate vectors that are nearly opposite. A value near 0 indicates that the vectors are independent of one another. Cosine similarity was also computed between the FC networks and 1000 anatomical null models. These null models were generated using the Brain Connectivity Toolbox (BCT; http://www.brain-connectivity-toolbox.net) by randomly rewiring the anatomical network while preserving its degree distribution (BCT function "randmio_dir").

Temporal stability for each connection was calculated by first crosscorrelating each FC time series with itself to produce a normalized autocorrelation coefficient function. The autocorrelation coefficient function was therefore equal to 1.0 at a time lag of zero and ranged from -1.0 to 1.0 at all other time lags. To compare autocorrelation coefficient functions across structural categories, autocorrelation coefficient functions were categorized according to the underlying anatomical connectivity (bidirectional, unidirectional, or no direct connectivity). The mean autocorrelation function was then computed for connections within each structural category. Statistical significance was determined using a bootstrap procedure (Efron and Tibshi- 

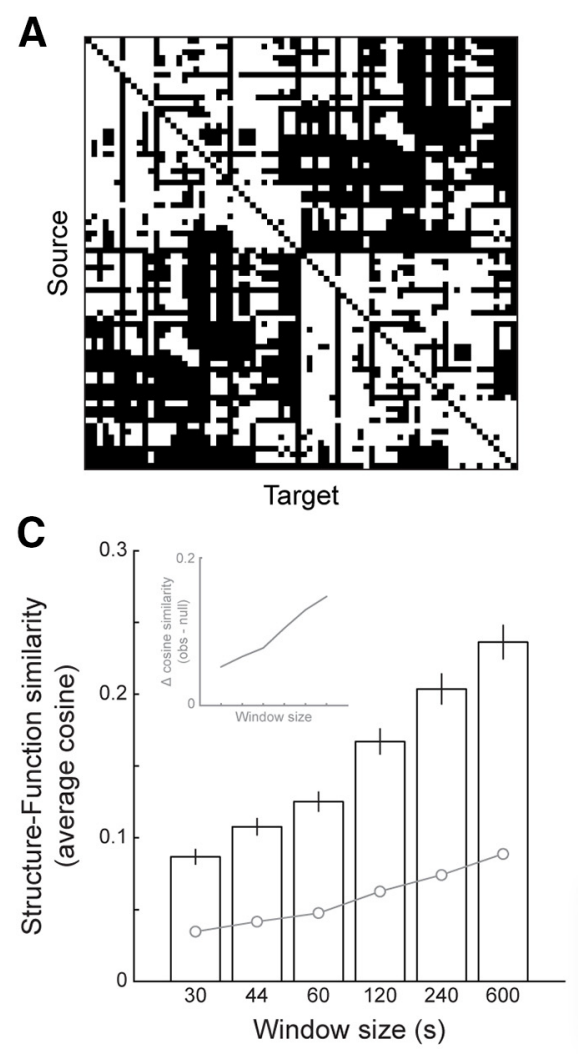

B

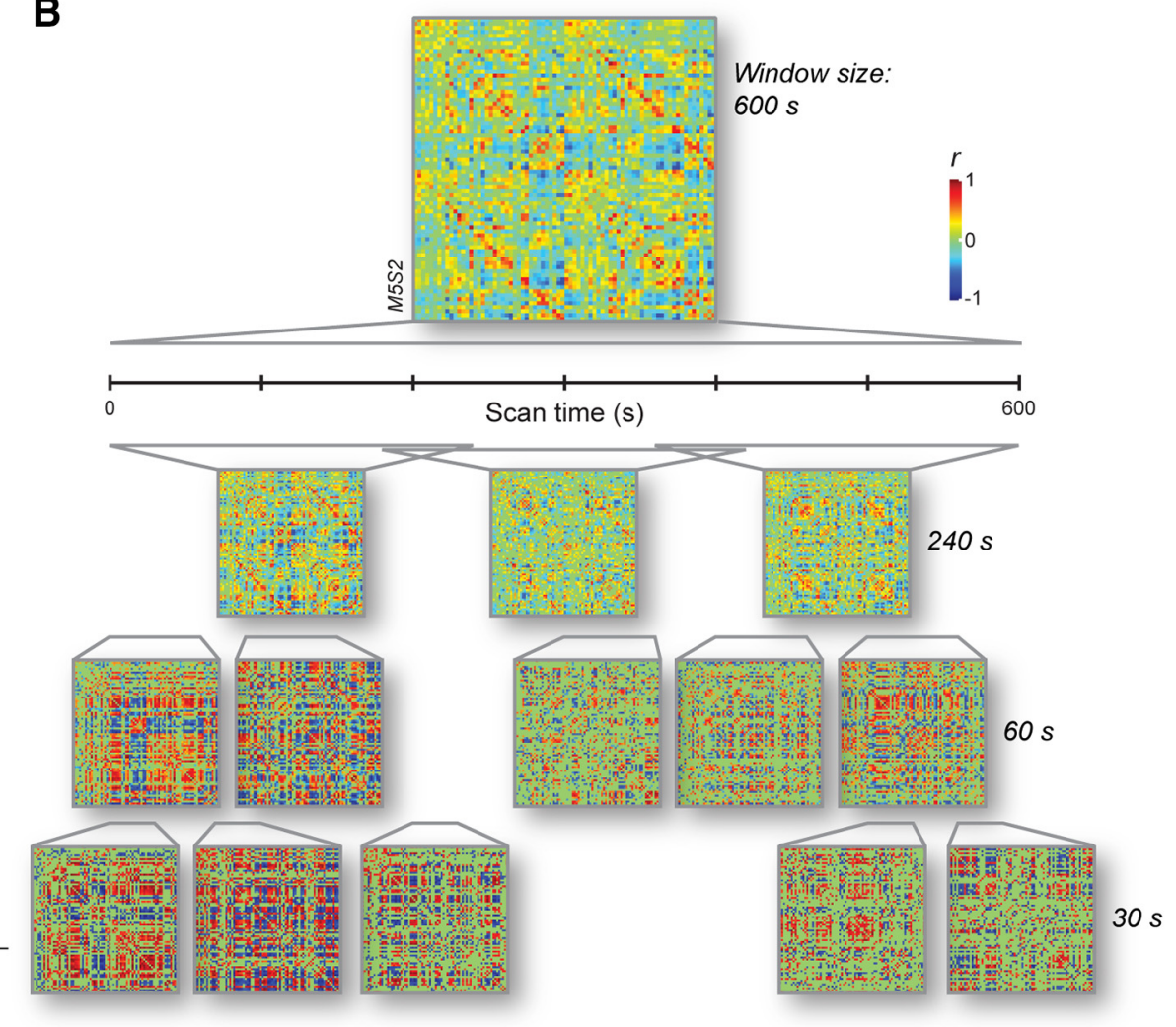

Figure 1. Connectivity matrices of macaque cortex. A, Structural connectivity matrix derived from the CoCoMac database. Connections are shown in white. $B$, Example of a "static" functional connectivity matrix (top) constructed from an entire resting-state BOLD-fMRI scan lasting 600 s from one animal. Examples of "dynamic" functional connectivity matrices constructed using different window sizes from the same scan (bottom). Functional connectivity was computed as Pearson correlations of ROl time series. Matrices are organized generally from anterior to posterior regions, for left then right hemispheres. Labels and ordering of regions within each hemisphere are specified in Table 1. C, Cosine similarity between rsFC and structural connectivity increases with increasing FC window size. Similarity was computed between the structural connectivity matrix and each rsFC matrix at each time point for all window sizes and averaged ( \pm SEM) across time points for each window size. For comparison with previous studies, performing this analysis using Pearson correlations resulted in a similar increase in correspondence between the structural and functional networks as window size increased ( $r$ range: $0.081-0.226$; Kruskal-Wallis, $p<0.001$ ). Gray circles represent the average \pm SEM of cosine similarity between windowed $F(n$ networks and 1000 null models with fixed degree distributions.

rani, 1993) to estimate $95 \%$ confidence intervals for the mean autocorrelation coefficient functions. We additionally computed a single measure of temporal stability for each functional connection by averaging its autocorrelation function across all lags. To ensure that the FC time series were stationary and that temporal stability could be inferred from their autocorrelation coefficient functions, we performed augmented DickeyFuller tests for unit roots (Said and Dickey, 1984) on the FC time series to determine whether they were nonstationary. For FC time series constructed with $30 \mathrm{~s}$ windows, nearly all (99.9\%) were not found to have unit roots and were therefore considered stationary. The proportion of stationary FC time series decreased somewhat as window size increased (44 s: 98.6\%; $60 \mathrm{~s}:$ $87.5 \% ; 120 \mathrm{~s}: 39.8 \% ; 240 \mathrm{~s}: 22.8 \%)$. Of note, the proportion of stationary FC time series did not differ across structural categories for time series constructed using 30,60,120, and $240 \mathrm{~s}$ window sizes (1-way ANOVA tests, all $p>0.235)$. For the $44 \mathrm{~s}$ window size, there were slightly fewer $(p<0.01)$ stationary FC time series for the bidirectional structural category $(97.7 \%)$ compared with the unidirectional $(99.1 \%)$ and no direct connectivity (99.4\%) categories.

Distances between ROIs were computed using the direct Euclidean distance between their centroids. A rich club organization of the macaque anatomical network was identified using the BCT (for details, see Harriger et al., 2012). This involved calculating the rich club coefficient (BCT function: "rich_club_bd") over the range of node degrees in the structural network. Coefficients were then normalized by dividing them by the average coefficient determined from 10,000 randomized networks with preserved degree distributions (BCT function: "makerandCIJdegreesfixed"). Statistical significance was determined by computing a onesided $p$-value using the null distribution of coefficients from the 10,000 randomized networks. An FDR procedure $(p<0.05)$ was then applied to correct for multiple comparisons.

Additional graph measures were computed to describe each node's functional embeddedness and the temporal variability of that embeddedness using the BCT. To determine overall functional embeddedness, we computed two measures of centrality (degree and betweenness) using the static rsFC networks. The degree of each node was calculated by taking the sum of its connection weights (BCT function "strengths_und"). The normalized betweenness of each node was calculated by taking the proportion of all shortest paths in the network that pass through that node (BCT function "betweenness_wei"). The variability of functional centrality was also determined by computing degree and betweenness for nodes in the dynamic rsFC networks and then calculating their SD over time for each node. However, interpreting variability measured by $\mathrm{SD}$ across a large range of centrality values is confounded by the fact that large magnitudes of centrality may naturally be associated with large SD. To account for the differences in functional centrality across nodes, we also computed the coefficient of variation $(\mathrm{CV}=\mathrm{SD} / \mathrm{mean})$ to obtain a normalized measure of dispersion. Degree, betweenness, $\mathrm{SD}$, and $\mathrm{CV}$ were all averaged across scans to obtain a single measure for each region in each window size.

\section{Results}

\section{Correspondence between anatomical connectivity and} dynamic rsFC

We derived dynamic resting-state cortical FC in macaques by performing regionwise correlations of BOLD time series for each scan across different window sizes using a sliding window ap- 
Table 1. Cortical ROIs and their abbreviations

\begin{tabular}{|c|c|}
\hline Abbreviation & ROI \\
\hline PFCpol & Prefrontal polar cortex \\
\hline $\mathrm{PFCm}$ & Medial prefrontal cortex \\
\hline PFCol & Orbitolateral prefrontal cortex \\
\hline PFCom & Orbitomedial prefrontal cortex \\
\hline PFCoi & Orbitoinferior prefrontal cortex \\
\hline PFCdl & Dorsolateral prefrontal cortex \\
\hline PFCdm & Dorsomedial prefrontal cortex \\
\hline $\mathrm{PFCl}$ & Centrolateral prefrontal cortex \\
\hline PFCvl & Ventrolateral prefrontal cortex \\
\hline $\mathrm{CCa}$ & Anterior cingulate cortex \\
\hline $\mathrm{CCs}$ & Subgenual cingulate cortex \\
\hline$C \mathrm{cp}$ & Posterior cingulate cortex \\
\hline $\mathrm{CCr}$ & Retrosplenial cingulate cortex \\
\hline FEF & Frontal eye field \\
\hline PMCvl & Ventrolateral premotor cortex \\
\hline PMCdl & Dorsolateral premotor cortex \\
\hline $\mathrm{PMCm}$ & Medial premotor cortex \\
\hline M1 & Primary motor cortex \\
\hline S1 & Primary somatosensory cortex \\
\hline S2 & Secondary somatosensory cortex \\
\hline G & Gustatory cortex \\
\hline la & Anterior insula \\
\hline Ip & Posterior insula \\
\hline $\mathrm{A} 1$ & Primary auditory cortex \\
\hline $\mathrm{A} 2$ & Secondary auditory cortex \\
\hline $\mathrm{HC}$ & Hippocampus \\
\hline PHC & Parahippocampal cortex \\
\hline $\mathrm{TCC}$ & Central temporal cortex \\
\hline TCs & Superior temporal cortex \\
\hline TCv & Ventral temporal cortex \\
\hline PCi & Inferior parietal cortex \\
\hline PCip & Intraparietal cortex \\
\hline $\mathrm{PCm}$ & Medial parietal cortex \\
\hline PCs & Superior parietal cortex \\
\hline VACv & Anterior visual area (ventral) \\
\hline VACd & Anterior visual area (dorsal) \\
\hline V2 & Visual area 2 \\
\hline V1 & Visual area 1 \\
\hline
\end{tabular}

proach. To examine the relationship between FC dynamics and the underlying anatomical architecture, we first computed the cosine similarity between each dynamic rsFC network and the structural connectivity network. Figure $1 C$ shows how the average similarity between the structural and functional networks increased significantly with increasing FC window size (KruskalWallis, $p<0.001)$. These cosine similarity values were significantly greater than those between the FC networks and anatomical null models (Wilcoxon rank sum tests, all $p<0.001$; Fig. 1C). Cosine similarity between windowed FC and null models increased somewhat with window size (Kruskal-Wallis, $p<$ 0.01 ), but the increase is substantially greater for the observed data than for the null models (Fig. 1C, inset). Together, these results suggest that functional networks constructed using lowfrequency dynamics best reflect the underlying anatomical structure. All proceeding data analyses will focus on dynamic rsFC computed from a sliding window size of $60 \mathrm{~s}$ because it represented an intermediate window size. Results from all other window sizes were similar and are presented as indicated.

\section{Temporal stability of rsFC is supported by local anatomical connectivity}

Consistent with previous reports (Hutchison et al., 2013b), we observed fluctuations in rsFC on the order of seconds to minutes
(Fig. $1 B$ ). Figure $2 A$ shows an example of how whole-brain rsFC patterns recapitulated over time within a single scanning session. To determine the extent to which each connection changes over time, we first computed a normalized autocorrelation coefficient function for each functional connection. The autocorrelation coefficient function describes how statistically correlated the FC at a single time point is with itself at all other time points in the FC time series. We therefore used the autocorrelation coefficient as a measure of the temporal stability of each functional connection. Although the use of autocorrelation in an absolute sense requires the time series be stationary, our main interest was the relative difference of the autocorrelation across functional connections and whether such differences were related to the underlying anatomical connections. We compared the autocorrelation coefficient functions for pairs of regions that were anatomically connected to those that were not anatomically connected. Figure $2 B$ illustrates how FC was significantly more correlated with itself across most lags (190/271, 70.1\%) for pairs of regions with reciprocal structural connections than for pairs that were not structurally connected. Results were similar for other window sizes (Fig. $2 C)$. The autocorrelation coefficient function for pairs with unidirectional structural connectivity was not on average significantly different from either reciprocally connected or unconnected pairs (Fig. $2 B, C)$, suggesting that the temporal stability for these region pairs fell somewhere in between.

We then averaged the autocorrelation coefficient function across all lags to generate a single temporal stability metric for each connection. It is important to note that the strength of static rsFC and the autocorrelation coefficient of the FC time series are not necessarily related. For example, a functional connection that is weak, but consistently so, would have high temporal stability. Mean temporal stability computed using a $60 \mathrm{~s}$ window was very weakly correlated with the distance between regions (Pearson correlation, $r=-0.05, p<0.001$ ), suggesting that the observed rsFC dynamics were not due to spatial blurring of the BOLD signal or other acquisition-related artifacts. Temporal stability was strongly correlated with the magnitude of static rsFC for both reciprocally connected $(r=0.70, p<0.001)$ and structurally unconnected pairs $(r=0.58, p<0.001)$. Static rsFC, however, is known to decrease with increasing distance between regions (Honey et al., 2009), so we regressed distance from the static rsFC strength to more accurately examine the relationship between static rsFC and dynamic rsFC. The residuals of the static FCdistance relationship were still well correlated with dynamic FC stability ( $r=0.66, p<0.001$; see Table 2 for other window sizes).

Figure 3 illustrates the most and least temporally stable dynamic FC for regions with and without structural connectivity. At a 1/16 threshold, there were more highly stable connections for structurally connected than unconnected pairs (Fig. 3A). The most stable FC of structurally connected pairs was concentrated between sensory (e.g., primary and secondary auditory, visual and somatosensory cortices), motor (e.g., primary and premotor cortices), and early association areas (e.g., visual association, posterior parietal and posterior cingulate cortices) (see Fig. $3 B, C$ for other thresholds). Many of the most stable functional connections between structurally connected pairs were between homologous cortical regions (Fig. 3, horizontal red lines). There were also many structurally connected regions with highly unstable FC. These were more widely distributed between regions of the prefrontal cortices, between prefrontal areas and sensory areas (e.g., between PFC and visual, auditory and somatosensory areas), as well as between prefrontal and temporal cortical areas. Moreover, some region pairs with no direct structural connec- 

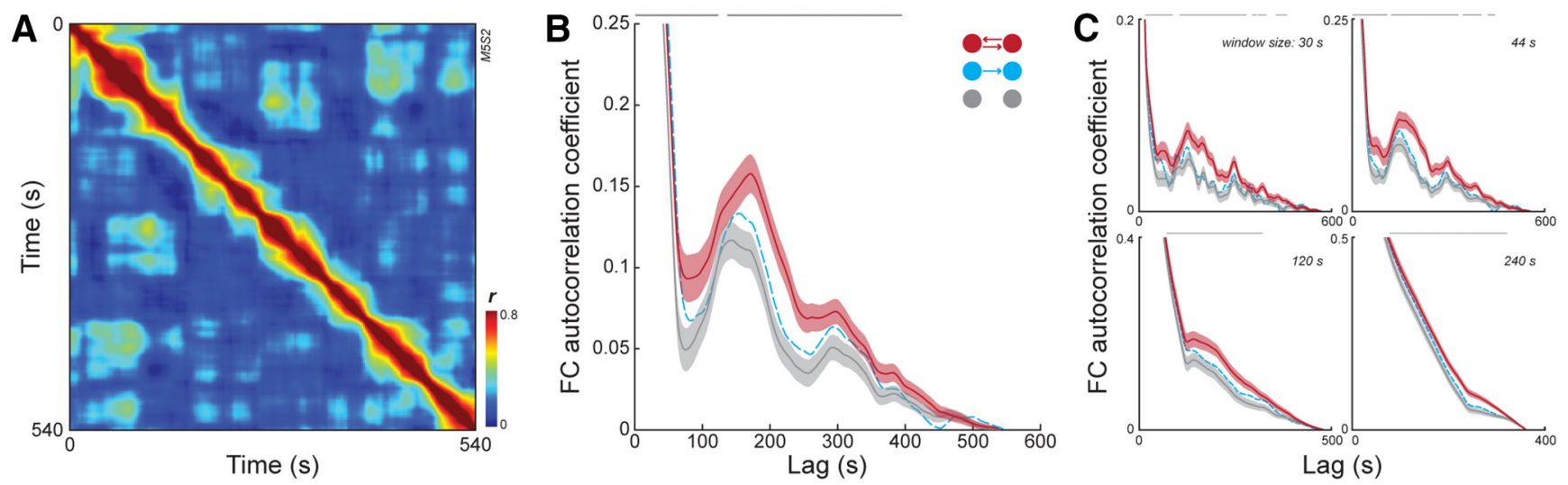

Figure 2. Stability of time-varying rsFC. $\boldsymbol{A}$, Whole-brain fluctuations in rsFC show repeated patterns across an example scanning session for a window size of 60 s. $\boldsymbol{B}$, Mean ( $\pm 95 \%$ Cl) autocorrelation coefficient function across all pairs of regions with reciprocal structural connectivity (red) and pairs with no structural connectivity (gray) for a window size of $60 \mathrm{~s}$. Mean function for pairs having unidirectional structural connectivity also shown (blue dashed). C, Mean $( \pm 95 \% \mathrm{Cl})$ autocorrelation coefficient functions for other window sizes. Lags with significant differences in autocorrelation coefficients between reciprocal and no structural connectivity classes denoted by gray points along the top.

Table 2. Temporal stability correlations at various window sizes

\begin{tabular}{lllll}
\hline & \multicolumn{4}{l}{ Window size $(\mathrm{s})$} \\
\cline { 2 - 5 } Temporal stability correlation with & 30 & 44 & 120 & 240 \\
\hline Euclidean distance & -0.06 & -0.05 & -0.05 & -0.06 \\
Static FC strength & & & & \\
$\quad$ Bidirectional SC & 0.71 & 0.71 & 0.63 & 0.52 \\
$\quad$ No direct SC & 0.51 & 0.57 & 0.56 & 0.50 \\
Residual of SC-distance regression & 0.65 & 0.67 & 0.60 & 0.50 \\
Number of intermediary regions & & & & \\
$\quad$ All pairs with no direct SC & 0.11 & 0.13 & 0.14 & 0.12 \\
$\quad$ Pairs with explicitly absent SC & $0.13(p=0.09)$ & $0.15(p<0.05)$ & 0.29 & 0.30 \\
\hline Correlation coefficients as specified, all $p<0.001$ unless otherwise specified. & &
\end{tabular}

tions still exhibited highly stable FC. These tended to be longrange functional connections between prefrontal areas and sensory areas. Similar observations of the distributions of stable and unstable FC across the human brain have recently been made (Gonzalez-Castillo et al., 2014; Zalesky et al., 2014).

The strength of static FC between regions that have no direct structural connectivity is known to increase with the number of other regions that are structurally connected to both (Adachi et al., 2012). We investigated whether these intermediary regions also played a role in resting-state functional dynamics. For the set of regions with no direct structural connectivity, temporal stability increased with an increasing number of intermediary regions that had a direct structural connection to both regions within the unconnected pair (Fig. 4; Pearson correlation, $r=0.14, p<$ 0.001; Table 2). This relationship was more pronounced when analysis was limited to only those pairs that were explicitly stated to be unconnected in CoCoMac $(r=0.23, p<0.01$; Table 2$)$. The dynamic nature of functional interactions was therefore also mediated by polysynaptic anatomical connections.

\section{Rich club structure confers functional stability}

A recent study in humans has shown how functional connections are more stable within modules than across modules (Zalesky et al., 2014). Using a previously described decomposition of our static functional network (Shen et al., 2012), we found similar results in our macaque dataset. Functional connections within modules had significantly greater temporal stability than connections across modules (Wilcoxon rank sum tests, $p<0.001$ ) for all but the largest window size (240 s, $p=0.43$ ).
Communication across modules is thought to proceed via functional hubs. Previous studies have suggested that hubs identified at coarse timescales are highly variable in their topological role at finer timescales (Honey et al., 2007; Mišić et al., 2011; Allen et al., 2014). To examine the extent to which the structural, topography affects these differences in functional dynamics, we first identified a structural "rich club" set of hubs. A rich club organization in which central regions were more densely interconnected than what is expected by chance was detected across a range of degrees (Fig. 5A). We examined two particular rich clubs, the first being the smallest subset of regions $\left(\mathrm{RC}_{\text {small }}\right.$, degree $\geq 101$ ) and the second being the subset most significantly different from the null distribution $\left(\mathrm{RC}_{\text {sig }}\right.$, degree $\left.\geq 91\right)$. $\mathrm{RC}_{\text {small }}$ consisted of 10 bilateral regions that included cingulate, parietal, and prefrontal areas and $\mathrm{RC}_{\text {sig }}$ also included the posterior insula, dorsolateral premotor cortex, and additional prefrontal regions (Fig. 5B). Functional connections were then classified according to this structural rich club organization to determine whether any differences exist in their temporal stability. Rich club connections were functional connections between two structural rich club nodes, feeder connections were those between a rich club node and a non-rich club node, and local connections were those between two non-rich club nodes (van den Heuvel et al., 2012). Note that all of these functional connections have underlying structural connectivity and only differ in terms of the structurally-determined topological role of their respective nodes. We considered the negative weights in functional networks to be meaningful and therefore generated graphs by taking the absolute value of rsFC weights. Graphs created by keeping only positive weights, as well as by thresholding at various levels, did not yield qualitatively different results (Table 3 ). For both the $\mathrm{RC}_{\text {small }}$ and $\mathrm{RC}_{\text {sig }}$ networks, temporal stability was significantly different across the three connection types (Kruskal-Wallis tests, both $p<0.001$; Fig. 5C,D). Somewhat surprisingly, a post hoc analysis revealed how rich club functional connections had significantly greater temporal stability than the other connection types (Tukey-Kramer method, $p<0.05$ ), whereas the stability of feeder and local connections were not significantly different from one another $(p>0.05)$. The strength of static rsFC within and outside of the rich club, however, did not follow this pattern, suggesting that topological differences in rsFC dynamics were not simply due to differences in static rsFC strength. For the $\mathrm{RC}_{\text {small }}$ network, the strength of rich club and local connections were not 
A
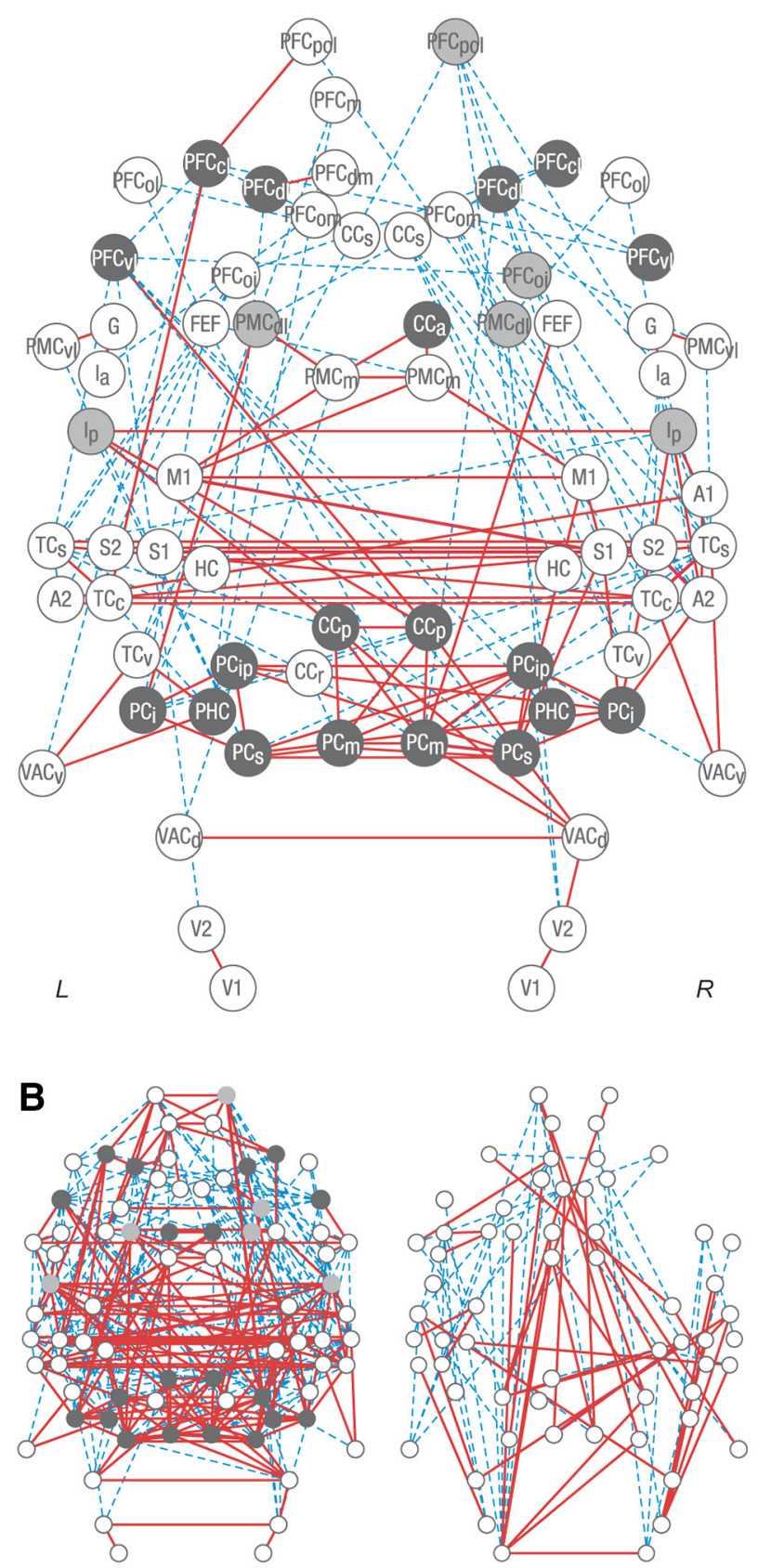

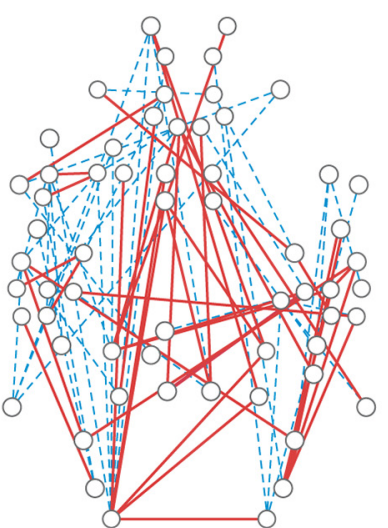

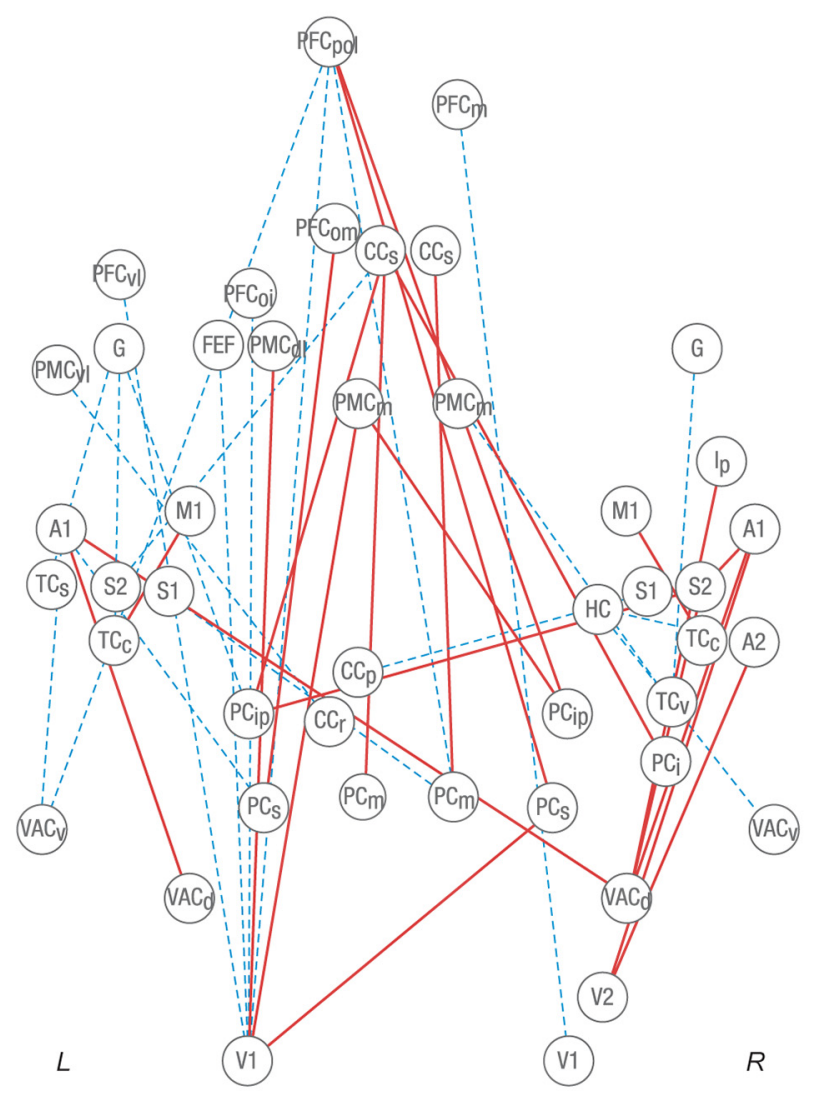

C

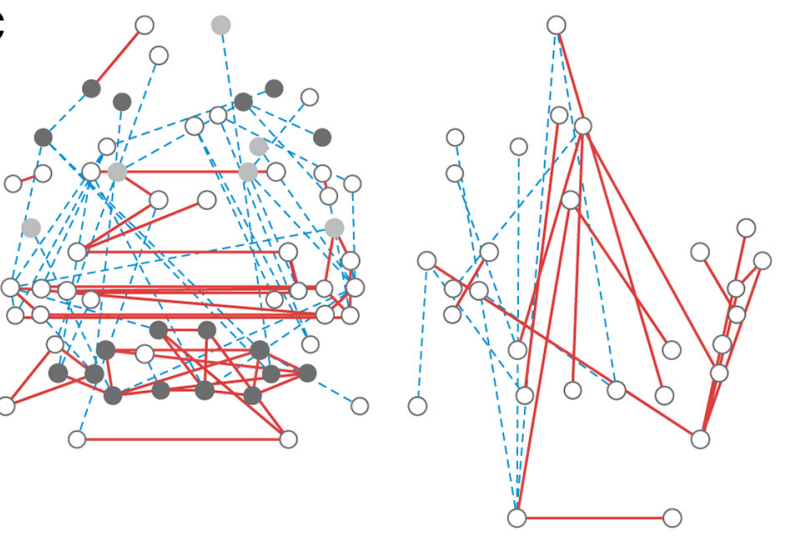

Figure 3. Most (red solid) and least (blue dashed) temporally stable rsFC connections for pairs with reciprocal structural connectivity (left) and pairs with no direct structural connectivity (right) for a window size of $60 \mathrm{~s}$. Graphs were thresholded to the top and bottom $1 / 16(\boldsymbol{A}), 1 / 8(\boldsymbol{B})$, and $1 / 32(\boldsymbol{C})$ of the overall distribution. Structurally identified rich club nodes for the smallest RC level $\left(\mathrm{RC}_{\text {small }}\right)$ detected are shown in dark gray and the additional $\mathrm{RC}$ nodes that contribute to the most significant $\mathrm{RC}$ level $\left(\mathrm{RC}_{\text {sig }}\right)$ detected are shown in light gray. Only pairs with explicitly absent connections (in at least one direction) are included. Nodes with no connections at these thresholds are not shown.

different from one another $(p>0.05)$ and for the $\mathrm{RC}_{\text {sig }}$ network, the strength of local connections were significantly greater than both rich club and feeder connections $(p<0.05$; Fig. $5 E)$. These results suggest that the functional interactions within the structural rich club occur more consistently over time than interactions outside of the structural rich club. Our findings further suggest that the brain's structural organization confers stability to otherwise highly flexible and dynamic functional networks.

These findings are somewhat paradoxical to previous studies that have reported how functional connections involving hubs are variable (Allen et al., 2014) and how functional hubs can have variable topological roles at finer timescales (Honey et al., 2007). To reconcile our findings with these previous studies, we first used graph theoretical tools to identify functional hubs by computing the degree and betweenness of each region in the static rsFC condition. We then related these static centrality measures to the variability of centrality measures computed using dynamic rsFC (cf. Honey et al., 2007). Consistent with previous findings, regions that were highly functionally embedded on average had variable functional roles over time. The SD of both functional degree and betweenness increased with increasing centrality (Pearson correlation, $r=0.48, p<0.001$ and $r=0.43, p<0.001$, 


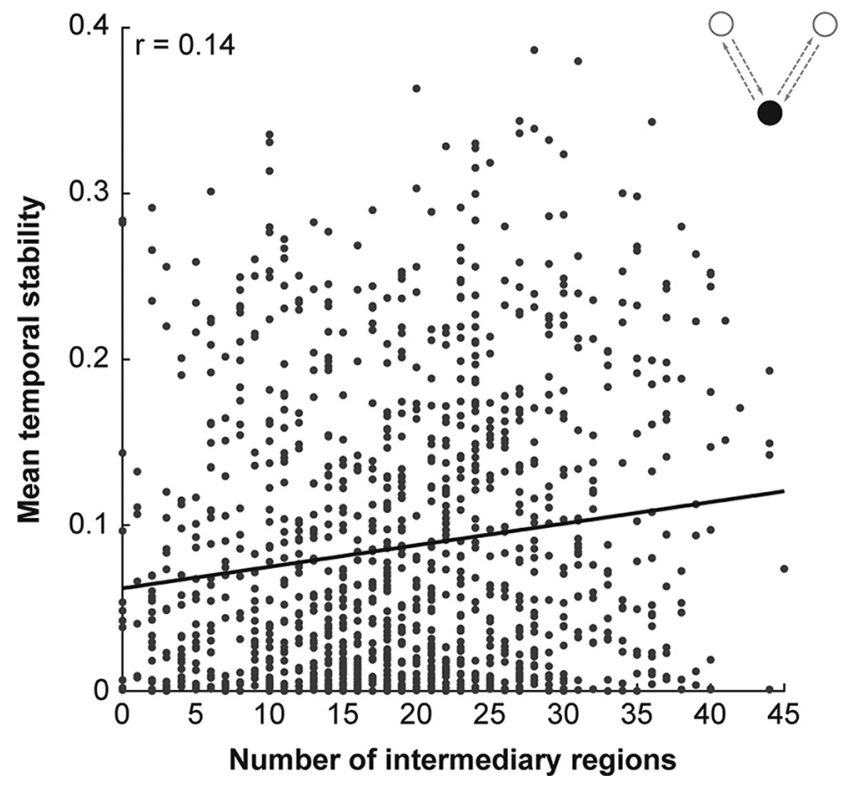

Figure 4. Temporal stability of region pairs having no direct structural connectivity as a function of the number of intermediary regions between them for a window size of $60 \mathrm{~s}$. See Table 2 for correlations at other window sizes.

respectively; Fig. 6A). This effect, however, was reversed when variability was normalized for differences in centrality across regions: The normalized variability $(\mathrm{CV})$ of both functional degree and betweenness decreased with increasing centrality $(r=-0.78$, $p<0.001$ and $r=-0.65, p<0.001$, respectively; Fig. $6 B$; see Table 3 for other graph thresholds and time windows). A particular set of functional hubs were also identified being a part of the structural rich club. These regions included the bilateral posterior cingulate cortex, the bilateral medial parietal cortex, the right intraparietal cortex, and the right posterior insula. This set of structure-function hubs were also among the regions that had the lowest degree and betweenness CVs (Fig. 6, filled colored circles). Our results suggest that, although hub regions are highly variable in their functional roles at finer timescales, they are not as variable as would be expected by their high centrality.

\section{Discussion}

In this study, we examined the extent to which the brain's anatomical organization influences the fluctuations observed in resting-state fMRI networks. We found that both local and global rsFC dynamic properties were bounded by structural connectivity across multiple timescales. Both direct and indirect structural connectivity was associated with greater temporal stability in rsFC. Despite their high variability in centrality over time, hub regions operated within a relatively small functional range. This was likely due to constraints imposed by the structural topology, with the greatest temporal stability occurring within the structurally defined rich club core.

Simulations of dynamic resting-state networks have suggested how the brain's underlying structure is best reflected at the coarsest timescales (Honey et al., 2007). We have provided an empirical demonstration of this relationship by showing how similarity between rsFC and structural connectivity increased with increasing $\mathrm{rsFC}$ window size. This relationship existed regardless of whether we used cosine or Pearson correlation methods to compute similarity. Together, these findings suggest that structural connectivity is expressed in rsFC networks at the lowest frequencies of BOLD fluctuations. At finer timescales, rsFC networks explore different functional configurations that do not reflect the entire structural network. However, even at these finer timescales, functional configurations are constrained by anatomical connectivity and likely reflect subsets of the network structure.

Our data add to a growing literature on the complex nature of the structure-function relationship (for review, see Damoiseaux and Greicius, 2009). Even at coarse timescales, rsFC is not a perfect representation of the underlying structural connectivity. In part, the disconnect between structure and function is due to the contribution of polysynaptic interactions to rsFC (Vincent et al., 2007; Honey et al., 2009; Adachi et al., 2012). We found that these structural constraints also extend to the dynamic properties of rsFC. Temporal stability increased with an increasing number of intermediary structural connections between otherwise unconnected regions. Functional changes that occur at the cellular level may also contribute to the lack of agreement between macroscale structure and function (Stephan et al., 2009; Friston, 2011). Short-term synaptic plasticity, for example, plays a significant role in shaping how information is processed within neural circuits (Abbott and Regehr, 2004). Neuromodulatory projections from the basal forebrain and thalamus are often excluded from macroscale studies, including ours, but have widespread effects on networks important for cognitive functions (Hasselmo and Sarter, 2011; Varela, 2014). The structure-function link also depends on the completeness of the structural dataset used. In our case, the binary nature of our structural connectivity matrix does not reflect the variability of connection weights (i.e., axonal fiber density) or distances (i.e., tract lengths) that exists across brain regions. These structural properties are known to play a role in static network organization (Ercsey-Ravasz et al., 2013) and may be particularly important in shaping functional dynamics. Including these features in future studies may help to inform the observed relationship between structure and function (Deco et al., 2014).

We replicated a recent finding that functional connections within modules are more stable over time than connections across modules (Zalesky et al., 2014) using a previously described decomposition of our static functional network. Importantly, we have previously shown how the modularity of that static functional network is supported by dense reciprocal anatomical projections within modules and sparse unidirectional anatomical connections across modules (Shen et al., 2012; Messé et al., 2015). Our current findings data further suggest that functional network dynamics are supported by the brain's underlying anatomical structure. We have additionally extended our understanding of the role that structural connectivity plays in long-range communication (i.e., across modules) by examining the functional variability of hub regions. Previous studies have suggested that measures of centrality, and functional hubs in particular, are highly variable (Honey et al., 2007; Mišić et al., 2011; Tagliazucchi et al., 2012; Allen et al., 2014; Yang et al., 2014). The variability of hub regions has implications for flexibility in cognitive function and behavior. For example, regional variability in functional topology during training predicts the extent to which participants can learn a motor task (Bassett et al., 2011), with the greatest temporal variability in heteromodal association regions of the prefrontal and temporal cortices (Bassett et al., 2013). These previous findings in both resting-state and task-based analyses indicating that integrative functional regions are highly variable seem inconsistent with the view that they are structurally connected and that structural connectivity constrains rsFC. Moreover, we identified a structural rich club in which rich club connections were found to exhibit significantly greater functional temporal stability than other kinds of connections. We reconciled these 
A

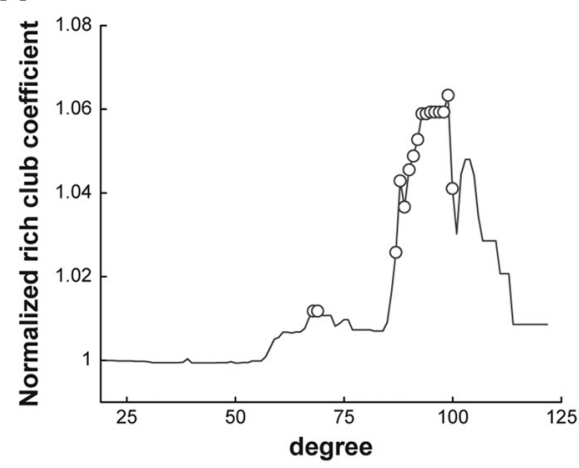

B

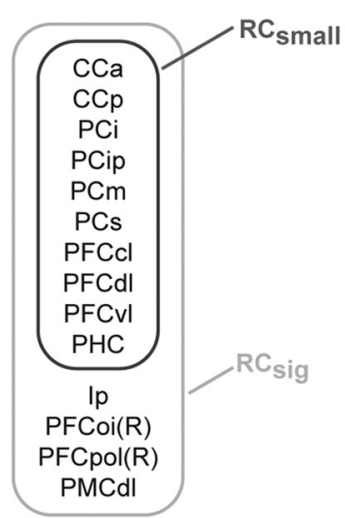

C

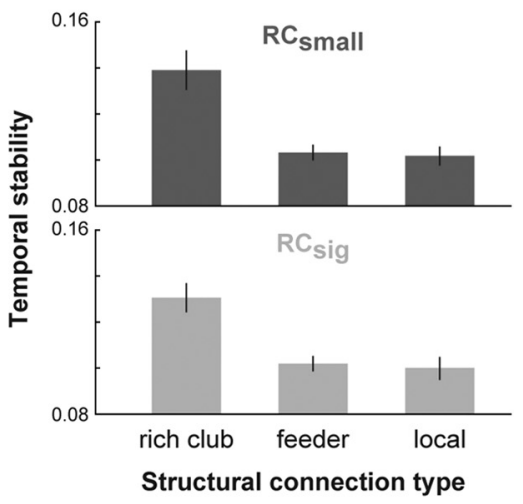

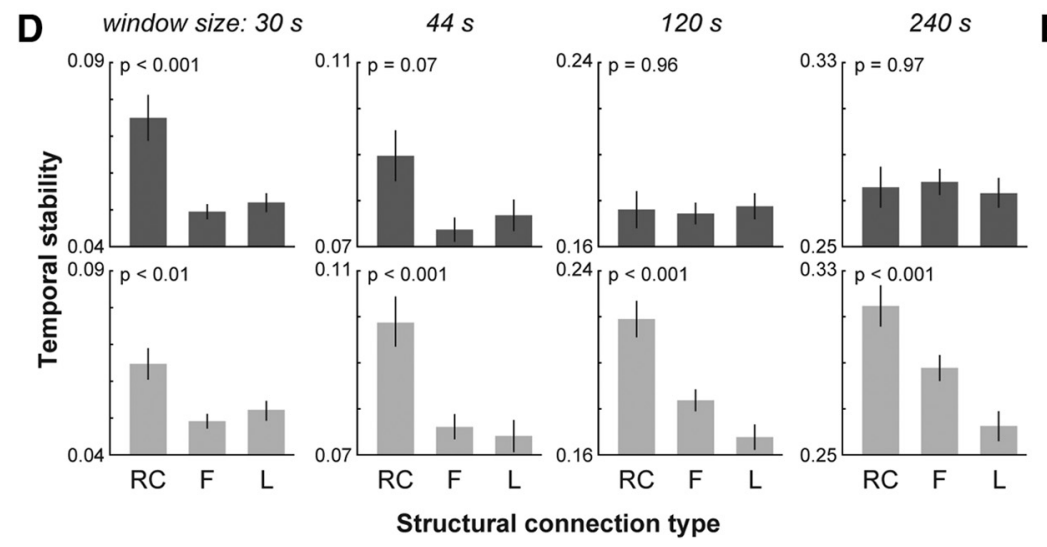

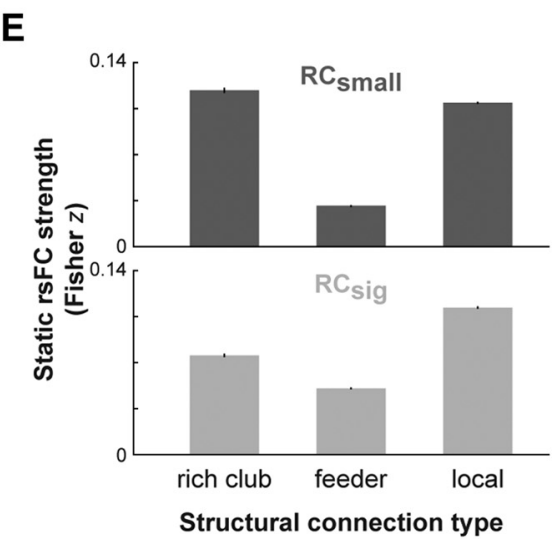

Figure 5. Macaque rich club structural network supports resting-state functional dynamics. $A$, Structural rich club network detection. The normalized rich club coefficient is plotted over the range of degrees. Circles denote coefficients significantly greater than those in randomized networks. $\boldsymbol{B}$, Regions comprising the smallest $\left(\mathrm{RC}_{\text {small }}\right)$ and the most significant structural rich clubs detected $\left(\mathrm{RC}_{\mathrm{sig}}\right)$. Regions are bilateral unless indicated. C, Mean ( \pm SEM) functional temporal stability for rich club, feeder, and local connections at a window size of $60 \mathrm{~s}$. D, Mean temporal stability at other window sizes as indicated. $\boldsymbol{E}$, Mean ( \pm SEM) rsFC strength of rich club, feeder, and local connections computed from the static condition.

A
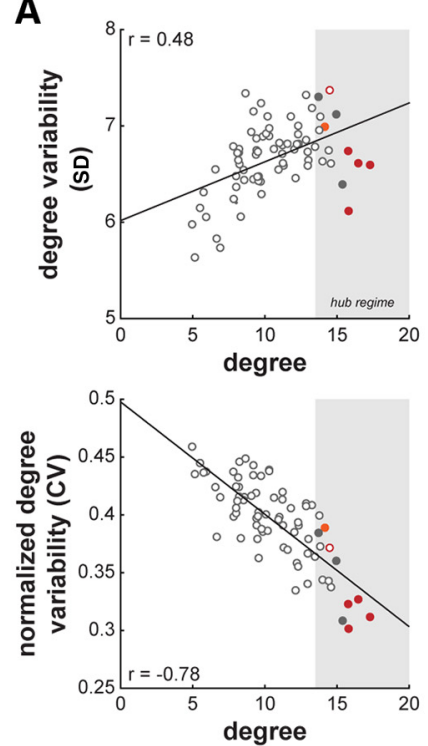

B
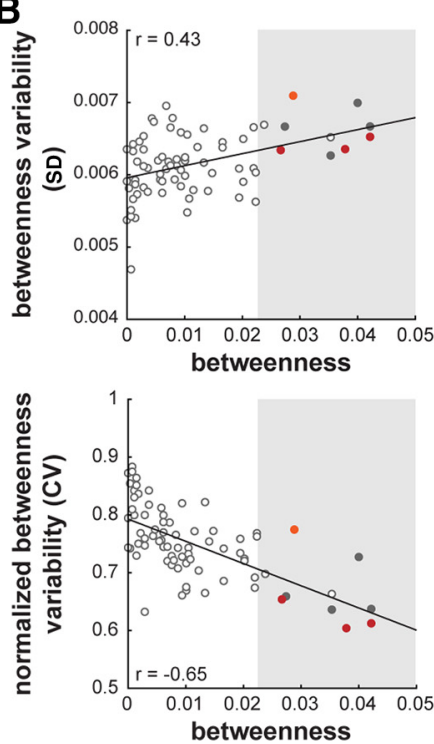

C

$\begin{array}{cc}\begin{array}{c}\text { Average } \\ \text { (static) }\end{array} & \text { Possible configurations } \\ \text { (dynamic) }\end{array}$
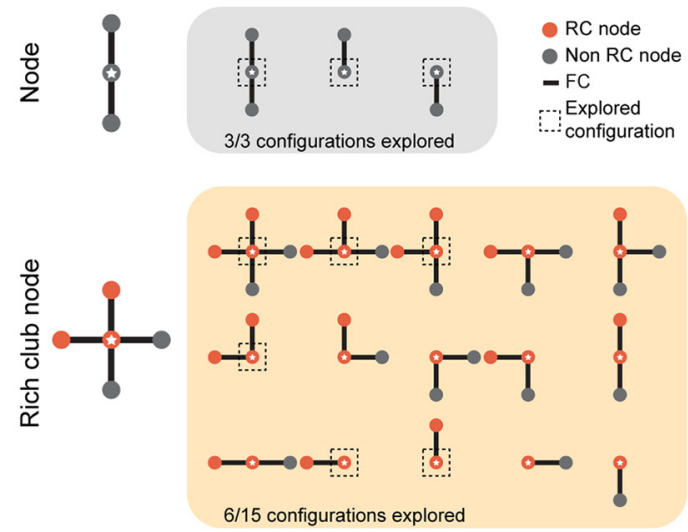

Figure 6. Variability (SD, top) of degree $(\boldsymbol{A})$ and betweenness $(\boldsymbol{B})$ increases with functional embeddedness. When normalized for differences in centrality across nodes, however, variability (CV, bottom) of degree and betweenness decreases with functional embeddedness at a window size of $60 \mathrm{~s}$. The range in which hubs were detected ( $>$ mean $+1 \mathrm{SD})$ for each centrality measure is denoted by the light gray region. Nodes identified as functional hubs using both centrality measures denoted by filled circles. Functional hubs that were additionally identified as structural rich club hubs denoted in red $\left(\mathrm{RC}_{\text {small }}\right)$ and orange $\left(\mathrm{RC}_{\text {sig }}\right)$. See Table 3 for other graph thresholds and window sizes. $C$, Schematic representation of the constraints structural connectivity can place on functional variability. Rich club hubs are highly functionally variable, but work well within theirfunctional range. Top, Examplenon-hub node and its possible dynamic configurations that can be explored. Bottom, Example rich club hub showing the range of possible configurations with the tendency to explore configurations that involve other rich club nodes that share strong and direct structural connections. 
Table 3. Variability (SD) and normalized variability (CV) of degree and betweenness correlations with functional embeddedness across various graph thresholds and window sizes

\begin{tabular}{|c|c|c|c|c|c|c|c|c|}
\hline & \multicolumn{4}{|c|}{ Threshold } & \multicolumn{4}{|l|}{ Window size (s) } \\
\hline & $60 \%$ & $40 \%$ & $20 \%$ & Positive weights only & 30 & 44 & 120 & 240 \\
\hline \multicolumn{9}{|l|}{ Variability (SD) } \\
\hline Betweenness & 0.42 & 0.38 & 0.45 & $0.26(p<0.05)$ & $0.19(p=0.10)$ & 0.46 & 0.58 & 0.61 \\
\hline \multicolumn{9}{|c|}{ Normalized variability (CV) } \\
\hline Degree & -0.77 & -0.83 & -0.88 & -0.86 & -0.65 & -0.73 & -0.81 & -0.84 \\
\hline Betweenness & -0.65 & -0.65 & -0.61 & -0.53 & -0.60 & -0.60 & -0.62 & -0.62 \\
\hline
\end{tabular}

Correlation coefficients as specified, all $p<0.001$ unless otherwise indicated.

seemingly paradoxical findings by first replicating previous findings that high functional variability is associated with high functional embedding. However, when we took into account the differences in range within which each region could operate by computing the $\mathrm{CV}$, we found the opposite relationship: $\mathrm{CV}$ decreased with increasing functional embeddedness. In particular, a set of structure-function hubs that included the posterior cingulate and medial parietal cortical areas had some of the lowest CVs. These areas correspond with hub regions that have the greatest functional variability as reported in previous human studies (Allen et al., 2014; Gonzalez-Castillo et al., 2014). These data suggest that, although hubs are flexible in function, they explore only a limited range of all possible configurations afforded by the available synaptic and polysynaptic pathways. In particular, rsFC dynamics are biased by the brain's rich club structure (Fig. 6C). That is, rich club nodes tend to be structurally connected to each other and that tendency dictates how consistently they interact with one another, as observed in the higher temporal stability of the rsFC dynamics. Related to this, a recent study suggested how the structural rich club might be responsible for integrative information processing across different resting-state networks (van den Heuvel and Sporns, 2013b). We add to this understanding by demonstrating that the structural rich club has direct consequences on resting-state dynamics.

Differences in observations of hub variability between the present study and previous ones in awake human subjects may also be attributed to our anesthetized "resting-state" approach. The low-dose of isoflurane used in the present study is known to produce robust and homologous FC patterns (for review, see Hutchison and Everling, 2012), as well as stable temporal FC patterns that suggest the preservation of intrinsic functional organization (Hutchison et al., 2014). Nevertheless, anesthesia has recently been shown to alter the temporal structure of restingstate networks and, at the highest levels of sedation, functional network dynamics are limited and instead reflect the rigid structure of the anatomical network (Barttfeld et al., 2015). Wakefulness may therefore be a less dynamically constrained "resting-state" and a direct comparison between existing human studies with resting-state dynamics in awake animals is needed in future studies.

Consideration of the structure-function relationship is incomplete without an understanding of the ongoing fluctuations in rsFC. In simulations, stationary models of rsFC based on the underlying structural connectivity still leave a large proportion of the variance in the structure-function relationship unexplained (Messé et al., 2014), suggesting that network structure may play a significant role in influencing the nonstationary properties of functional networks. A recent study has shown how the reconstruction of large-scale structural connectivity from rsFC is most successful when the dynamical system is at a critical point just before instability (Deco et al., 2014). In simulations, simply al- lowing both high- and low-activity patterns to exist at the level of local populations (i.e., nodes) is enough to result in the emergence of realistic whole-brain FC dynamics from an otherwise fixed structural network (Hansen et al., 2015). Together, these simulation data suggested that the structural network allows for maximal variability of resting-state networks while restraining their dynamics from entering an unstable state. Our observations that functional hubs work well within their available range and that the densely structurally connected rich club regions were the most temporally stable support this view. These findings show how the brain's structural architecture sets the constraints for the range of functional network configurations.

\section{References}

Abbott LF, Regehr WG (2004) Synaptic computation. Nature 431:796-803. CrossRef Medline

Adachi Y, Osada T, Sporns O, Watanabe T, Matsui T, Miyamoto K, Miyashita Y (2012) Functional connectivity between anatomically unconnected areas is shaped by collective network-level effects in the macaque cortex. Cereb Cortex 22:1586-1592. CrossRef Medline

Allen EA, Damaraju E, Plis SM, Erhardt EB, Eichele T, Calhoun VD (2014) Tracking whole-brain connectivity dynamics in the resting state. Cereb Cortex 24:663-676. CrossRef Medline

Bakker R, Wachtler T, Diesmann M (2012) CoCoMac 2.0 and the future of tract-tracing databases. Front Neuroinform 6:30. Medline

Barttfeld P, Uhrig L, Sitt JD, Sigman M, Jarraya B, Dehaene S (2015) Signature of consciousness in the dynamics of resting-state brain activity. Proc Natl Acad Sci U S A 112:887-892. CrossRef Medline

Bassett DS, Wymbs NF, Porter MA, Mucha PJ, Carlson JM, Grafton ST (2011) Dynamic reconfiguration of human brain networks during learning. Proc Natl Acad Sci U S A 108:7641-7646. CrossRef Medline

Bassett DS, Wymbs NF, Rombach MP, Porter MA, Mucha PJ, Grafton ST (2013) Task-based core-periphery organization of human brain dynamics. PLoS Comput Biol 9:e1003171. CrossRef Medline

Benjamini Y, Krieger A, Yekutieli D (2006) Adaptive linear step-up procedures that control the false discovery rate. Biometrika:491-507.

Bezgin G, Vakorin VA, van Opstal AJ, McIntosh AR, Bakker R (2012) Hundreds of brain maps in one atlas: registering coordinate-independent primate neuro-anatomical data to a standard brain. Neuroimage 62:67-76. CrossRef Medline

Bressler SL, Kelso JA (2001) Cortical coordination dynamics and cognition. Trends Cogn Sci 5:26-36.

Damoiseaux JS, Greicius MD (2009) Greater than the sum of its parts: a review of studies combining structural connectivity and resting-state functional connectivity. Brain Struct Funct 213:525-533. CrossRef Medline

Deco G, Jirsa VK, McIntosh AR (2011) Emerging concepts for the dynamical organization of resting-state activity in the brain. Nat Rev Neurosci 12:43-56. CrossRef Medline

Deco G, Jirsa VK, McIntosh AR (2013) Resting brains never rest: computational insights into potential cognitive architectures. Trends Neurosci 36:268-274. CrossRef Medline

Deco G, McIntosh AR, Shen K, Hutchison RM, Menon RS, Everling S, Hagmann P, Jirsa VK (2014) Identification of optimal structural connectivity using functional connectivity and neural modeling. J Neurosci 34 : 7910-7916. CrossRef Medline 
Efron B, Tibshirani R (1993) An introduction to the bootstrap. New York: Chapman and Hill.

Eger EI 2nd (1984) The pharmacology of isoflurane. Br J Anaesth 56:71S99S. Medline

Ercsey-Ravasz M, Markov NT, Lamy C, Van Essen DC, Knoblauch K, Toroczkai Z, Kennedy H (2013) A predictive network model of cerebral cortical connectivity based on a distance rule. Neuron 80:184-197. CrossRef Medline

Friston KJ (2011) Functional and effective connectivity: a review. Brain Connect 1:13-36. CrossRef Medline

Gonzalez-Castillo J, Handwerker DA, Robinson ME, Hoy CW, Buchanan LC, Saad ZS, Bandettini PA (2014) The spatial structure of resting state connectivity stability on the scale of minutes. Front Neurosci 8:138. Medline

Greicius MD, Supekar K, Menon V, Dougherty RF (2009) Resting-state functional connectivity reflects structural connectivity in the default mode network. Cereb Cortex 19:72-78. CrossRef Medline

Hagmann P, Cammoun L, Gigandet X, Meuli R, Honey CJ, Wedeen VJ, Sporns O (2008) Mapping the structural core of human cerebral cortex. PLoS Biol 6:e159. CrossRef Medline

Hansen EC, Battaglia D, Spiegler A, Deco G, Jirsa VK (2015) Functional connectivity dynamics: modeling the switching behavior of the resting state. Neuroimage 105:525-535. CrossRef Medline

Harriger L, van den Heuvel MP, Sporns O (2012) Rich club organization of macaque cerebral cortex and its role in network communication. PLoS One 7:e46497. CrossRef Medline

Hasselmo ME, Sarter M (2011) Modes and models of forebrain cholinergic neuromodulation of cognition. Neuropsychopharmacology 36:52-73. CrossRef Medline

Hermundstad AM, Bassett DS, Brown KS, Aminoff EM, Clewett D, Freeman S, Frithsen A, Johnson A, Tipper CM, Miller MB, Grafton ST, Carlson JM (2013) Structural foundations of resting-state and task-based functional connectivity in the human brain. Proc Natl Acad Sci U S A 110:61696174. CrossRef Medline

Honey CJ, Sporns O, Cammoun L, Gigandet X, Thiran JP, Meuli R, Hagmann P (2009) Predicting human resting-state functional connectivity from structural connectivity. Proc Natl Acad Sci U S A 106:2035-2040. CrossRef Medline

Honey CJ, Kötter R, Breakspear M, Sporns O (2007) Network structure of cerebral cortex shapes functional connectivity on multiple time scales. Proc Natl Acad Sci U S A 104:10240-10245. CrossRef Medline

Hutchison RM, Everling S (2012) Monkey in the middle: why non-human primates are needed to bridge the gap in resting-state investigations. Front Neuroanat 6:29. Medline

Hutchison RM, Leung LS, Mirsattari SM, Gati JS, Menon RS, Everling S (2011) Resting-state networks in the macaque at 7 T. Neuroimage 56: 1546-1555. CrossRef Medline

Hutchison RM, Womelsdorf T, Allen EA, Bandettini PA, Calhoun VD, Corbetta M, Della Penna S, Duyn JH, Glover GH, Gonzalez-Castillo J, Handwerker DA, Keilholz S, Kiviniemi V, Leopold DA, de Pasquale F, Sporns O, Walter M, Chang C (2013a) Dynamic functional connectivity: promise, issues, and interpretations. Neuroimage 80:360-378. CrossRef Medline

Hutchison RM, Womelsdorf T, Gati JS, Everling S, Menon RS (2013b) Resting-state networks show dynamic functional connectivity in awake humans and anesthetized macaques. Hum Brain Mapp 34:2154-2177. CrossRef Medline

Hutchison RM, Hutchison M, Manning KY, Menon RS, Everling S (2014) Isoflurane induces dose-dependent alterations in the cortical connectivity profiles and dynamic properties of the brain's functional architecture. Hum Brain Mapp 35:5754-5775. CrossRef Medline

Kötter R, Wanke E (2005) Mapping brains without coordinates. Philos Trans R Soc Lond B Biol Sci 360:751-766. CrossRef Medline

Li CX, Patel S, Auerbach EJ, Zhang X (2013) Dose-dependent effect of isoflurane on regional cerebral blood flow in anesthetized macaque monkeys. Neurosci Lett 541:58-62. CrossRef Medline

Markov NT, Ercsey-Ravasz MM, Ribeiro Gomes AR, Lamy C, Magrou L, Vezoli J, Misery P, Falchier A, Quilodran R, Gariel MA, Sallet J, Gamanut R, Huissoud C, Clavagnier S, Giroud P, Sappey-Marinier D, Barone P, Dehay C, Toroczkai Z, Knoblauch K, et al. (2014) A weighted and directed interareal connectivity matrix for macaque cerebral cortex. Cereb Cortex 24:17-36. CrossRef Medline

Mars RB, Jbabdi S, Sallet J, O'Reilly JX, Croxson PL, Olivier E, Noonan MP, Bergmann C, Mitchell AS, Baxter MG, Behrens TE, Johansen-Berg H, Tomassini V, Miller KL, Rushworth MF (2011) Diffusion-weighted imaging tractography-based parcellation of the human parietal cortex and comparison with human and macaque resting-state functional connectivity. J Neurosci 31:4087-4100. CrossRef Medline

Masamoto K, Kanno I (2012) Anesthesia and the quantitative evaluation of neurovascular coupling. J Cereb Blood Flow Metab 32:1233-1247. CrossRef Medline

Messé A, Rudrauf D, Benali H, Marrelec G (2014) Relating structure and function in the human brain: relative contributions of anatomy, stationary dynamics, and non-stationarities. PLoS Comput Biol 10:e1003530. CrossRef Medline

Messé A, Hütt MT, König P, Hilgetag CC (2015) A closer look at the apparent correlation of structural and functional connectivity in excitable neural networks. Sci Rep 5:7870. CrossRef Medline

Mišić B, Vakorin VA, Paus T, McIntosh AR (2011) Functional embedding predicts the variability of neural activity. Front Syst Neurosci 5:90. Medline

Modha DS, Singh R (2010) Network architecture of the long-distance pathways in the macaque brain. Proc Natl Acad Sci U S A 107:13485-13490. CrossRef Medline

Pinneo LR (1966) On noise in the nervous system. Psychol Rev 73:242-247. CrossRef Medline

Rabinovich M, Varona P, Friston K (2012) Principles of brain dynamics: Global state interactions. Cambridge, Mass.: MIT.

Said S, Dickey D (1984) Testing for unit roots in autoregressive-moving average models of unknown order. Biometrika 71:599-607. CrossRef

Shen K, Bezgin G, Hutchison RM, Gati JS, Menon RS, Everling S, McIntosh AR (2012) Information processing architecture of functionally defined clusters in the macaque cortex. J Neurosci 32:17465-17476. CrossRef Medline

Shmuel A, Leopold DA (2008) Neuronal correlates of spontaneous fluctuations in fMRI signals in monkey visual cortex: Implications for functional connectivity at rest. Hum Brain Mapp 29:751-761. CrossRef Medline

Stephan KE, Kamper L, Bozkurt A, Burns GA, Young MP, Kötter R (2001) Advanced database methodology for the Collation of Connectivity data on the Macaque brain (CoCoMac). Philos Trans R Soc Lond B Biol Sci 356:1159-1186. CrossRef Medline

Stephan KE, Tittgemeyer M, Knösche TR, Moran RJ, Friston KJ (2009) Tractography-based priors for dynamic causal models. Neuroimage 47: 1628-1638. CrossRef Medline

Tagliazucchi E, von Wegner F, Morzelewski A, Brodbeck V, Laufs H (2012) Dynamic BOLD functional connectivity in humans and its electrophysiological correlates. Front Hum Neurosci 6:339. Medline

Teichert T, Grinband J, Hirsch J, Ferrera VP (2010) Effects of heartbeat and respiration on macaque fMRI: Implications for functional connectivity. Neuropsychologia 48:1886-1894. CrossRef Medline

Tinker JH, Sharbrough FW, Michenfelder JD (1977) Anterior shift of the dominant EEG rhythm during anesthesia in the Java monkey: correlation with anesthetic potency. Anesthesiology 46:252-259. CrossRef Medline

van den Heuvel MP, Sporns O (2013a) Network hubs in the human brain. Trends Cogn Sci 17:683-696. CrossRef Medline

van den Heuvel MP, Sporns O (2013b) An anatomical substrate for integration among functional networks in human cortex. J Neurosci 33:1448914500. CrossRef Medline

van den Heuvel MP, Mandl RC, Kahn RS, Hulshoff Pol HE (2009) Functionally linked resting-state networks reflect the underlying structural connectivity architecture of the human brain. Hum Brain Mapp 30:31273141. CrossRef Medline

van den Heuvel MP, Kahn RS, Goñi J, Sporns O (2012) High-cost, highcapacity backbone for global brain communication. Proc Natl Acad Sci U S A 109:11372-11377. CrossRef Medline

Van Essen DC (2004) Surface-based approaches to spatial localization and registration in primate cerebral cortex. Neuroimage 23:S97-S107. CrossRef Medline

Van Essen D, Drury H, Dickson J, Harwell J, Hanlon D, Anderson C (2001) An integrated software suite for surface-based analyses of cerebral cortex. J Am Med Informatics Assoc 8:443-459. CrossRef

Varela C (2014) Thalamic neuromodulation and its implications for executive networks. Front Neural Circuits 8:69. Medline

Vincent JL, Patel GH, Fox MD, Snyder AZ, Baker JT, Van Essen DC, Zempel JM, Snyder LH, Corbetta M, Raichle ME (2007) Intrinsic functional architecture in the anaesthetized monkey brain. Nature 447:83-86. CrossRef Medline

Yang Z, Craddock RC, Margulies DS, Yan CG, Milham MP (2014) Common intrinsic connectivity states among posteromedial cortex subdivisions: Insights from analysis of temporal dynamics. Neuroimage 93:124-137. CrossRef Medline

Zalesky A, Fornito A, Cocchi L, Gollo LL, Breakspear M (2014) Timeresolved resting-state brain networks. Proc Natl Acad Sci U S A 